\title{
The Latest Experiences Using A Cryogen Free Microcalorimeter Energy Dispersive X-Ray Spectrometer
}

\author{
Del Redfern", Joe Nicolosi*, Jens Höhne ${ }^{* *}$, Rainer Weiland ${ }^{* * *}$, \\ Birgit Simmnacher $^{* * *}$ and Christian Hollerith ${ }^{* * * *}$ \\ *EDAX Inc. Mahwah, NJ, USA \\ ** CSP Cryogenic Spectrometers GmbH, Munich, Germany \\ ***Infineon Technologies AG, Munich, Germany \\ ****Technische Universität München
}

The Polaris Microcalorimeter Energy Dispersive X-Ray Spectrometer has been in use at Infineon Technologies AG, Munich since April 2001. The microcalorimeter is presently in use as an every day tool, producing resolutions $<15 \mathrm{eV}$ at $1.5 \mathrm{keV}$.

This paper will discuss the first year of operation, review the goals of the project and how they have been met and review potential opportunities of this exciting new technology. The acceptance criteria that will be discussed include energy resolution, count rate and temperature hold time, all being required for suitable analysis of microelectronic devices.

The microcalorimeter is being used for routine analysis, including Ti/TiN, WSi2, $\mathrm{Si} / \mathrm{Ta} / \mathrm{W}$ and boron, phosphorus, silicon glass (BPSG) samples. Data will be presented showing the capabilities of the system to perform simple qualitative analysis on these materials. Small volume analysis is also regularly performed and data from thin layers, such as $\mathrm{SrBi} 2 \mathrm{Ta} 2 \mathrm{O} 9 / \mathrm{Pt} /$ and small particles will also be presented.

There are two main areas of concern to develop the microcalorimeter energy dispersive $\mathrm{x}$-ray spectrometer into a commercial product; the count rates (acquisition time) and vibration. At present the acquisition time for a spectrum to ensure the scientist is fully confident of the results is between 3 to 5 minutes. This paper will describe the actions proposed and undertaken to bring the acquisition time down to the more acceptable levels of around 60 seconds. Images at 100,000x magnification have shown little effect of vibration with the microcalorimeter running under normal operation. The target for vibration free operation is 200,000x; the work to reduce the level of vibration will be discussed.

Another development area to improve the suitability of the Polaris for industrial applications is the hold time (working time) of the adiabatic demagnetization refrigerator (ADR). The present beta unit has a hold time of approximately 8 hours before the ADR has to be recharged. A new ADR using the extended hold time unit is being evaluated, this would increase this hold time to over 24 hours and the outcome of the evaluation will be described in the paper. 

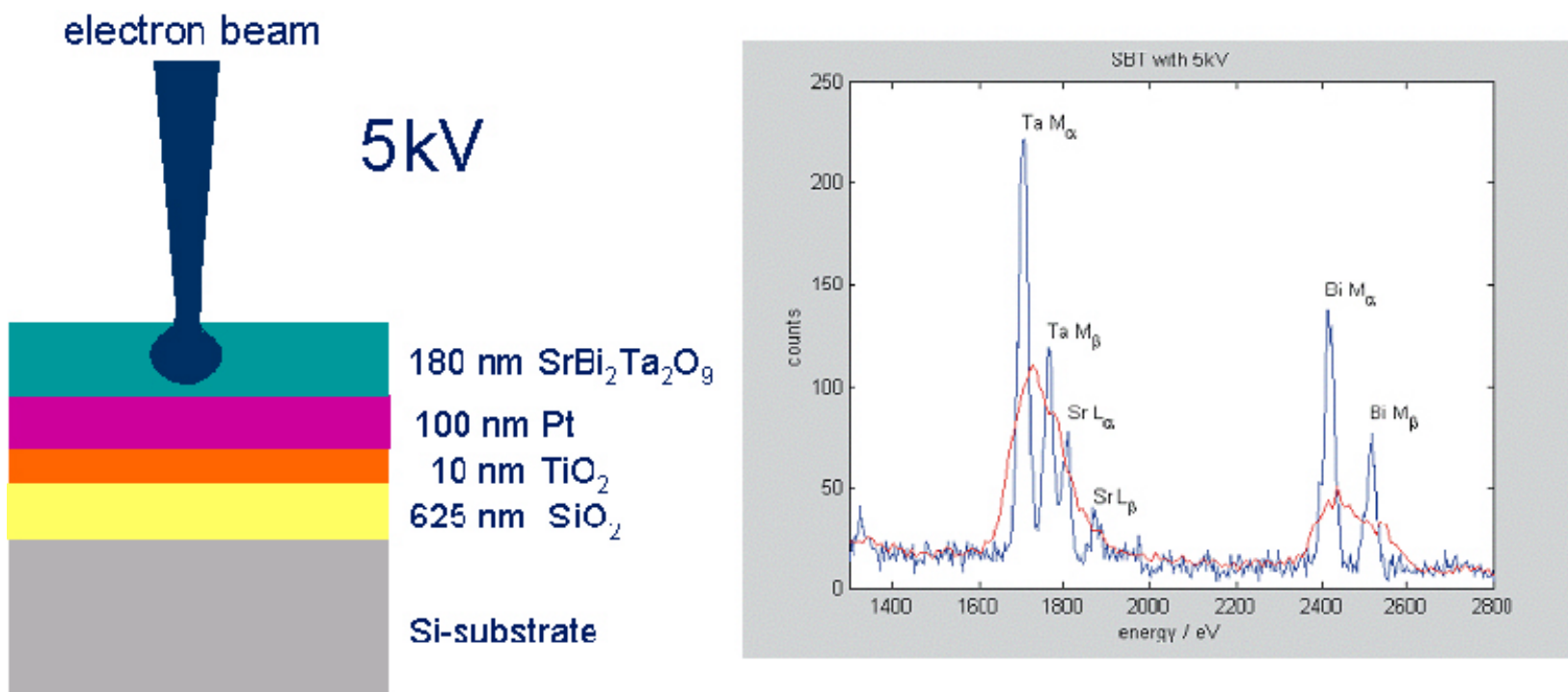

Spectra from the Microcalorimeter Energy Dispersive X-Ray Spectrometer of a layered sample using $5 \mathrm{kV}$ excitation voltage.

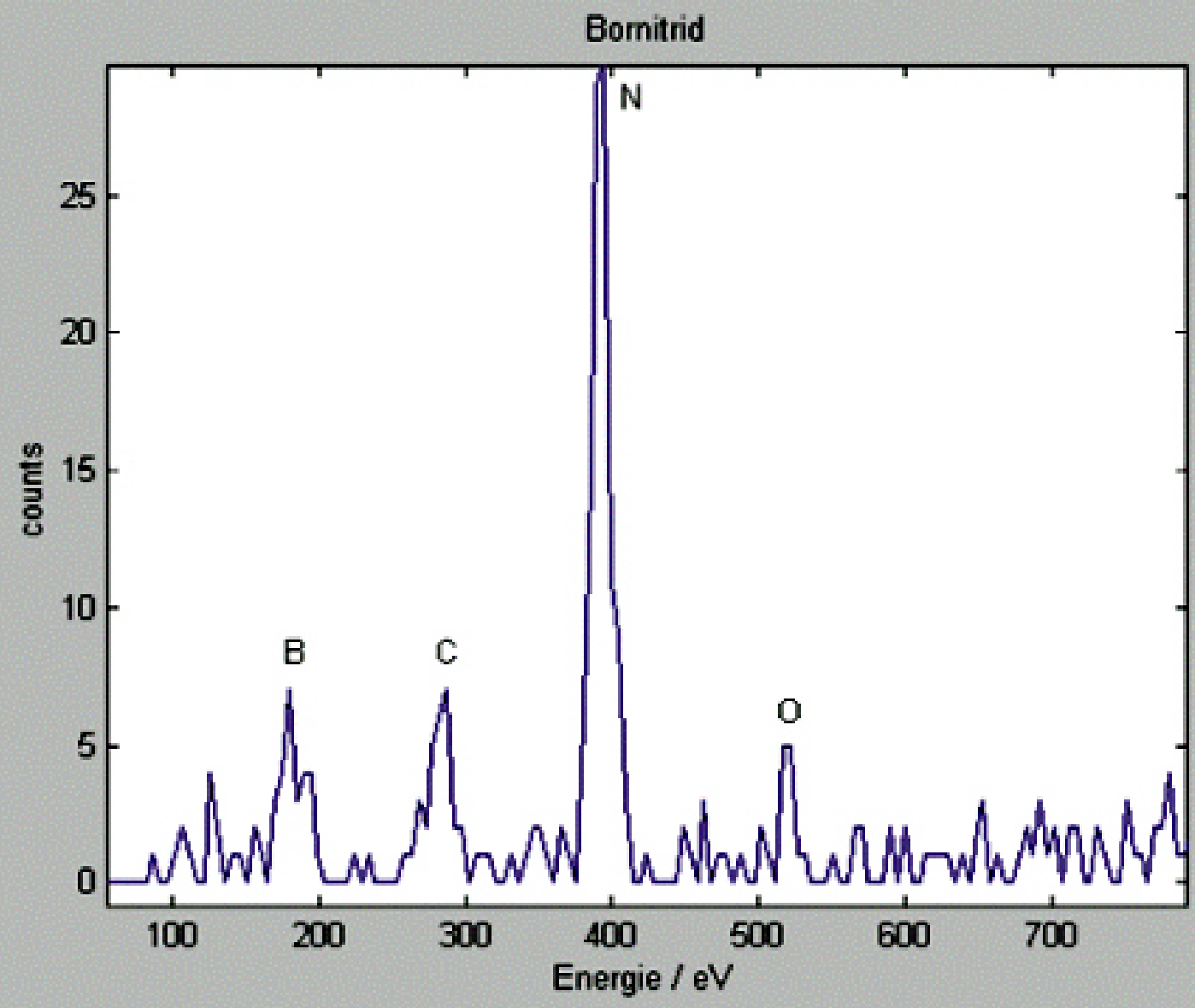

Spectra of a BN sample acquired using the Microcalorimeter EDS system 